\title{
Ueber das Verhalten von unterphosphorigsaurem Calcium im thierischen Körper.
}

\author{
Von \\ Dr. Theodor Panzer, Assistent. \\ Mittheilung aus dem Universitätslaboratorium für medicinische Chemie \\ in $W$ ien.
}

Wenn es sich um den chemischen Nachweis einer Phosphorvergiftung: handelt, und elementarer Phosphor in den Untersuchungsgegenständen nicht aufgefunden wird, so pflegt man, wie bekannt, nach phosphoriger Säure zu suchen. Hierzu bedient man sich in der Regel des Dusart-Blondlot'schen Verfahrens. Nun hat Dusart ${ }^{1}$ ) schon nachgewiesen, dass die unterphosphorige Säure bei Anwendung seiner Methode dieselben Erscheinungen zeigt, wie die phosphorige Säure. Die unterphosphorigsauren Salze werden aber zu therapeutischen Zwecken verwendet und deshalb ist, wenn in einem gegebenen Falle die Dusart-Blondlot'sche Methode ein positives Ergebniss liefert, nicht ohne Weiteres der Rückschluss auf die Anwesenheit von phosphoriger Säure, bezw. auf eine Phosphorvergiftung gestattet, sondern es muss immer noch die Möglichkeit in Betracht gezogen werden, dass dem betreffenden Individuum Hypophosphite einverleibt wurden. Es schien daher wünschenswerth, einige Aufschlïsse darüber zu erlangen, wie sich unterphosphorigsaure Salze im thierischen Organismus verhalten.

Schon Blondlot ${ }^{2}$ ) und später Paquelin und Joly ${ }^{3}$ ) fanden, dass, wenn Thieren ein Hypophosphit einverleibt wird, dieses im Harne wieder erscheint. Mir kam es hauptsächlich darauf an, die Frage zu beantworten, ob und wie lange diese Salze, wenn sie dem Thierkörper einverleibt werden, darin verbleiben, namentlich aber, ob sie dann in den Organen nachzuweisen sind. Zu diesem Zwecke wurden einige Versuche an Hunden ausgeführt, wobei das Calciumsalz der unterphosphorigen Sïure in Anwendung kam.

Ein 10,1 kg schwerer, männlicher Hund erhielt am 17. April um $11 \mathrm{Uhr}$ vormittags $1 \mathrm{~g}$ Calciumbypophosphit per os. Der im Verlaufe der folgenden 6 Stunden entleerte Harn (etwa $100 \mathrm{ccm}$ ) wurde gesammelt, in einen Wasserstoffentwickelungsapparat gebracht, in welchem verdünnte Schwefelsäure auf Zink wirkte, und das entweichende Gas durch Silbernitratlösung geleitet. Der in dieser Lösung entstandene schwarzbraune Niederschlag wurde durch Dekantation mit Wasser gewaschen und in einem zweiten, ganz kleinen Wasserstoffentwickelungsapparat geprüft, der so eingerichtet war, dass das entweichende Gas über mit Kalilange getränkte Bimssteinstückchen strich und dann aus einem Porzellanröhrchen entwich, woselbst es angezündet wurde. Es brannte mit stark

\footnotetext{
1) Compt. rend. 48, 1126.

2) Compt. rend. 52, 1197.

3) Compt. rend. 86, 1505.
} 
grüner Flamme, womit Phosphorwasserstoff, bezw. unterphosphorige Säure nachgewiesen war. Selbstrerständlich waren alle verwendeten Reagentien vorher auf ihre Reinheit geprüft worden.

Der bis zum nächsten Morgen (18. April, 9 Lhr frïh) entleerte Harn (etwa $100 \mathrm{ccm}$ ) nach demselben Terfahren verarbeitet, lieferte ebenfalls eine stark grüngefärbte Flamme, während der bis zum Morgen des 19. April entleerte, sowie die Harne der beiden darauffolgenden Tage keine Reaktion mehr zeigten.

Die Ausscheidung des Hypophosphits durch den Harn war daher mindestens nach 22 Stunden beendet. Im zu wissen, nach welcher Zeit dieses Salz bereits im Harne auftritt, wurde demselben Hunde am 22. April abermals $1 \mathrm{~g}$ unterphosphorigsaures Calcium verabreicht und in Zwischenräumen ron je einer halben Stunde Harn durch den Katheter entnommen. Schon der nach der ersten halben Stunde gewonnene Harn zeigte deutlich die Reaktion der unterphosphorigen Säure.

Dieser Versuch wurde nach einigen Tagen (am 25. April) an demselben Thiere wiederholt, nachdem rorher, um ganz sicber zu sein, nachgewiesen worden war, dass der Harn wieder frei von Hypophosphit war. Nur wurde diesmal dem Hunde in Zwischenräumen von je einer Viertelstunde der Harn mittels Katheters entnommen. Auch der nach der ersten Viertelstunde gewonnene Harn gab schon, zwar nicht stark, aber doch deutlich die Grünfärbung der Flamme. In dem am nächsten Tage nach der Einrerleibung des Hrpophosphits entleerten Kothe war keine unterphosphorige Säure nachzuweisen.

Am 30. April bekam der Hund wieder $1 \mathrm{~g}$ Calciumhypophosphit und wurde 6 Stunden darauf durch Verbluten aus der Carotis getödtet. Das Blut, sowie die wichtigsten Organe, nämlich der ganze Magendarmkanal, die Leber, beide Nieren und das Gehirn, wurden auf unterphosphorige Säure untersucht. Zu diesem Zwecke wurden die Organe zerkleinert - der Magen und Darm, nachdem daraus der Inhalt mechanisch entfernt worden war - mit Wasser übergossen und über Nacht bei Zimmertemperatur stehen gelassen. Am folgenden Tage wurde filtrirt und die so erhaltenen Flïssigkeiten wurden in gleicher Weise verarbeitet, wie dies bereits beim Harne beschrieben worden ist. Weder im Blute, noch in einem der Organe lonnte unterphosphorige Säure nachgewiesen werden mit Ausnahme des Magens und Darmes Diese lieferten nämlich eine deutliche Grüntärbung der Wasserstoff-Flamme.

Nach diesem auffallenden Ergebnisse musste der zuletzt angefürte Versuch an einem zweiten Hunde wiederholt werden. Einem $7.4 \mathrm{lig}$ schweren, männlichen Hunde wurde wieder $1 \mathrm{~g}$ Calciumbypophosphit in den Magen gebracht. Auch hier konnte bereits nach einer Viertelstunde im Harne unterphosphorige Säure nachgewiesen werden. Der Hund wurde diesmal schon 3 Stunden nach der Einverleibung des Salzes durch Verbluten aus der Carotis getödtet. Zugleich wurde der Harn aus der Blase entnommen und auf unterphosphorige Säure geprüft: er lieferte eine sehr starke Grïnfärbung der Wasserstoff-Flamme. 
Von den Leichentheilen wurden wieder Blut, Leber, beide Nieren, Gehirn und Magendarmkanal verarbeitet, doch wurde letzterer zur vollständigen Entfernung seines Inhaltes durch einen kräftigen Wasserstrabl ausgespült. Ferner wurden die zerkleinerten Organe mit Wasser auf dem Wasserbade erwärmt.

Diesmal konnte in keinem der Untersuchungsstücke unterphosphorige Säure nachgewiesen werden. Bei der Untersuchung der Nieren and des Blutes wurde eine ganz schwache Grüfärbung der Wasserstoff-Flamme beobachtet, aber die Erscheinung war doch nicht deutlich genug, um einen sicheren Schluss zuzulassen.

Um auch einige Aufschlïsse über die Ausscheidungsverhältnisse dieses Salzes beim Menschen zu erhalten, nahm ich selbst $1 \mathrm{~g}$ Calciumhypophosphit, das ich, nebenbei bemerkt, ohne Beschwerden vertrug. Nach einer Viertelstunde fand sich noch keine unterphosphorige Säure im Harne, wohl aber nach einer halben Stunde. Der Harn des folgenden Tages enthielt noch Hypophosphit, erst am zweitfolgenden Tage war der Harn frei davon. Die Ausscheidung scheint also beim Menschen etwas langsamer vor sich zu gehen.

Aus den beschriebenen Versuchen ergiebt sich also, dass einem Hunde einverleibtes unterphosphorigsaures Calcium rasch und recht vollständig resorbirt wird, den Organismus durchwandert, ohne irgendwo zurückgehalten zu werden, und sehr rasch wieder ausgeschieden wird. Die Ausscheidung dürfte jedenfalls innerhalb 24 Stunden beendet sein. Die Thatsache, dass in den Organen kein Hypophosphit gefunden wurde, möchte ich nicht so deuten, als ob wirklich keines darin vorhanden gewesen wäre, sondern ich möchte meinen, dass das DusartBlondlot'sche Verfahren zu wenig empfindlich ist, um die geringen, in den Organen enthaltenen Spuren von unterphosphoriger Säure nachzuweisen. Die Wasserstoff-Flamme zeigt allerdings die kleinsten Spuren von beigemengtem Phosphorwasserstoff an, es muss also die geringe Empfindlichkeit des Verfahrens in der Gewinnung des Phosphorsilberniederschlages zu suchen sein. Dass beim ersten Versuche im Magen und Darme unterphosphorige Säure gefunden wurde, scheint darauf zurückzuführen zu sein, dass Reste von Darminhalt mitverarbeitet wurden.

Für die forensische Praxis lassen sich nun aus diesen Versuchen folgende Regeln ableiten:

Kommt eine Phosphorvergiftung in Frage, und lässt es sich nachweisen dass die angeblich vergiftete Person auch nur einige Tage vor ilurem Tode kein Hypophosphit genommen hat, so braucht auf die unterphosphorige Säure überhaupt keine Rücksicht genommen zu werden.

Lässt sich dies aber nicht mit Sicherheit feststellen, so sind von der Untersuchung auf phosphorige Säure der Inhali des Magendarmkanales und der Harn auszuschliessen, dann wird, wenn wirklich ein Hypophosphit genommen wurde, dies das Ergebniss der Untersuchung nicht beeinträchtigen; denn es dürfte wohl der Schluss gerechtfertigt sein, dass, wenn die in den Organen eines so kleinen Thieres, wie die beiden Versuchshunde, nach Einverleibung der ver- 
hältnissmässig grossen Menge von $1 \mathrm{~g}$ vorhandenen Spuren ron unterphosphoriger Säure nicht mehr nacbgewiesen werden konnten, dies noch weniger der Fall sein wird, wenn dem bedeutend grösseren Organismus eines Menschen die weitaus geringeren medicinalen Gaben dieser Salze zugeführt werden.

\section{Referate.}

\section{Allgemeine analytische Methoden und Apparate.}

Zur Berechnung der Atomgwichte. - Zeitschr. angew. Chem. 1901, 14, $182-184$.

Einige zur Atomgewichtskommission delegirte Mitglieder des Vereins deutscher Chemiker haben an die Docenten der Chemie an den Hochschulen in Deutschland, Oesterreich und der Schweiz einen Aufruf gerichtet, welcher zur Beantwortung folgender Fragen auffordert: 1. Soll die Wasserstoffeinheit al: Grundlage zur Berechnung der Atomgewichte beibehalten werden? 2. Sollen die Atomgewichte grleichmässig mit 2 Decimalen angegeben werden, wobei die unsicheren Stellen durch den Druck zu kennzeichnen sind? 3. Soll die internationale Atomgewichtskommision auf dieser Grundlage die fortlaufende Bearbeitung der Atomgerwichtstabelle veranlassen? Von den eingelaufenen Antworten stimmen 19 für $0=16,103$ für $\mathrm{H}=1$.

Die engere Atomgewichtskommission der Deutschen Chemischen Gesellschaft hat dieser Kundgebung insofern Rechnung getragen, als sie in ihren Berichten 2 Tabellen veröffentlicht hat, von denen eine, die ,internationale" auf $0=16$ berechnet ist, während die zweite, die „didaktische" diese Zahlen im Verhältniss von 16:15,88 umrechnet, also auf $\mathrm{H}=1$ bezogen ist. (Vergl. diese Zeitschrift 1901, 4, 2053.)

J. Mayrhojer.

Otto Nasse: Ceber die Verwendbarkeit des Millon', chen Reagens. Pflüger's Archiv 1901, 83, 361-368.

Verf, verwendet an Stelle des Merkurinitrats eine wässerige Lösung von Queck. silberacetat, der einige Tropfen einer $1 \%$-igen Lösung ron Natrium- oder Kaliumnitrit zugesetzt sind. Meist genügt die saure Reaktion des Quecksilberacetats zum Eintritt der Färbung. Anderenfalls wird etwas verdünnte Essigwäure zugefügt. Die Salpetersäure ist demnach vollkommen unnöthig, damit wird auch die Ansicht hinfällig, dass es sich bei der Millon'schen Rerktion um eine Nitrirung bandle. Diese ist vielmehr zu den Nitroso-Reaktionen zu rechnen. Die Reaktion wird gehemmt durch Wasserstoffsuperoxyd, durch einen grösseren Gebalt des Reaktion:gemizches an Chloriden sowie auch durch Alkohol, der aber nach dem Erkalten ohne Sehaden zugefügt werden kann. Das Millon'sche Reagens, wie es Verf. rerwendet, färbt bei den einfach hydroxylirten Benzolderivaten und bei denen, in welchen nur noch ein Wasserstoffatom substituirt ist, die o-Verbindungen braunroth, die $\mathrm{p}$-Terbindungen blauroth bis blau, in starker Verdünnung rosaroth, die m-Verbindungen in wenig bestimmter Farbenschattirung. Die blauviolette p-Reaktion geben unter anderem auch die Eiweisskörper und deren Spaltungsprodukt, das Tyrosin, sowie von dessen Abkömmlingen das p-Kresol. Auch giebt fast jedes Phenol und jede Salicrlsäure die p-Reaktion, dank dem Gehalt von $\mathrm{p}$-Kresol und p-Oxybenzëosäure. Das Reagens ermöglicht es 